\title{
A NOTE ON UNITS IN SUPER-CYCLIC FIELDS
}

\author{
BY H. S. VANDIVER
}

1. Comparison of Two Known Results Concerning Cyclotomic Units. Kummer* first showed that if

$$
\zeta=e^{2 i \pi / l}
$$

with $l$ an odd prime, and if $\eta$ is a unit in $k(\zeta)$ such that

$$
\eta \equiv a(\bmod l)
$$

where $a$ is a rational integer, then

$$
\eta=\rho^{l},
$$

where $\rho$ is in $k(\zeta)$, provided none of the Bernoulli numbers

$$
B_{1}, B_{2}, \cdots, B_{d}, \quad(d=(l-3) / 2),
$$

is divisible by $l$. Kummer's proof of this depended on the fact that under the assumptions mentioned there exists an integer $c$ prime to $l$ such that

$$
\eta^{c}=E_{1}^{a_{1}} E_{2}{ }^{a_{2}} \cdots E_{d}^{a_{d}} .
$$

Here

$$
\begin{aligned}
E_{n} & =\prod_{i=0}^{d} \epsilon\left(\zeta^{r i}\right)^{r-2 i n}, \\
\epsilon & =\left(\frac{\left(1-\zeta^{r}\right)\left(1-\zeta^{-r}\right)}{(1-\zeta)\left(1-\zeta^{-1}\right)}\right)^{1 / 2} .
\end{aligned}
$$

From this we obtain an identity in an indeterminate $x$ by adding a certain multiple of

$$
\frac{x^{l}-1}{x-1}
$$

Setting $x=e^{v}$, taking logarithms and differentiating $2 n$ times, $(n=1,2, \cdots, d)$, we find, using relations in another paper, $\dagger$

* Journal für Mathematik, vol. 40 (1850), p. 128.

$\dagger$ Transactions of this Society, vol. 31 (1929), pp. 619-620, relations (4) and (5). 


$$
a_{1} \equiv a_{2} \cdots \equiv a_{d} \equiv 0(\bmod l),
$$

which is the result. if

By using a quite different method, Hilbert* gave proof that

$$
\eta \equiv 1\left(\bmod \lambda^{l}\right), \quad \lambda=1-\zeta
$$

and $k(\zeta)$ is a regular field, then $\eta=\rho^{l}$.

A field $k(\zeta)$ is said to be regular if and only if $l$ is prime to its class number. It is known that this condition is equivalent to the statement that the set (1) contains no numbers divisible by $l$.

Comparing the different forms of $\eta$ in the two statements of Kummer and Hilbert, we note that if $\eta=a+\theta l$, where $\theta$ is in $k(\zeta)$, we may write $\theta=b+\lambda \theta_{1}$ where $b$ is rational, and obtain $\eta=a+l b+\lambda^{l} \omega$. Now $(a+l b)$ is not necessarily equal to 1 , so the two forms are not the same.

Hilbert's proof of the result as stated by him depended on his theory of class-fields. It was reproduced by Landau $\dagger$ who commented on the great length of the proof and the complexity of one of the lemmas involved, that is, the existence of a system of relative fundamental units in a Kummer field.

In the present paper I shall consider further the principles involved in the demonstration of this theorem and give an extension of it involving super-cyclic fields. I shall also consider analogous questions in connection with the cyclotomic field which is not regular. The proofs, in the main, will be merely sketched.

2. A Theorem Concerning Primary Units in Super-Cyclic Tields. Furtwängler $\ddagger$ gave the result that if $K$ contains the field $k(\zeta)$ and if the class number of $K$ be $H=l^{h} q, q \neq 0(\bmod l)$, and a basis for the Abelian group formed by the $q$ th powers of the ideal classes of $K$ be $C_{1}, C_{2}, \cdots, C_{e}$, then there exists a basis for the singular primary numbers in $K, \omega_{1}, \omega_{2}, \cdots, \omega_{e}$, such that any singular primary number in $K$ may be written in the

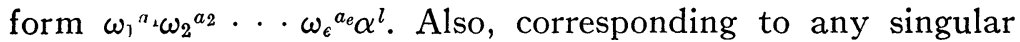
primary number belonging to the basis, there is an ideal class $C$ belonging to the basis of the so-called irregular class group.

* Werke, vol. 1, p. 287.

$\dagger$ Vorlesungen über Zahlentheorie, vol. 3, p. 240, p. 258.

$\ddagger$ Mathematische Annalen, vol. 43 (1907), p. 18. 
The above shows that if we have the primary units in $K$, that is, a unit $\eta$ such that

$$
\eta \equiv \gamma^{l}\left(\bmod \lambda^{l}\right),
$$

it follows that if the field $K$ has a class number which is prime to $l$, then no $C$ exists and therefore no singular primary number. Hence $\eta$ is an $l$ th power in $K$.

The above argument can be put in somewhat different form by employing the law of reciprocity

$$
\left\{\frac{\alpha}{\beta}\right\}=\left\{\frac{\beta}{\alpha}\right\},
$$

where each member denotes an $l$ th power character in $K$ and $\alpha$ is a primary integer in $K$. As a special case of this we have*

$$
\left\{\frac{\omega}{\beta}\right\}=1,
$$

where $\omega$ is a singular primary number in $K$. Let $\beta=\mathfrak{p}^{h}$, where $\mathfrak{p}$ is a prime ideal in $K$ and $h$ is the class number of $K$; then the above relation gives

$$
\left\{\frac{\omega}{p}\right\}=1
$$

for any $p$ in $K$ prime to $l$. From this it follows $\dagger$ that $\omega$ is the $l$ th power of the number in $K$; whence $\eta$ is also an $l$ th power. We may then state the following theorem.

If an algebraic field $K$ contains a cyclotomic field $k(\zeta), \zeta=e^{2 i \pi / l}$, and $\eta$ is a primary unit in the former field, then $\eta$ is the lth power of a unit in $K$ provided the class number of $K$ is prime to $l$.

We now observe that super-cyclic fields exist in which the class number is prime to $l$. Such a field is a Kummer field defined by $\zeta$ and $(\sigma)^{1 / l}$, where $\sigma$ is a unit in $k(\zeta)$ which is not primary. The class number of such a field is prime to $l$, provided $\ddagger$ the class number of $k(\zeta)$ is prime to $l$.

- Takagi, Journal of the College of Sciences, Tokyo, vel. 44 (1922), p. 26.

$\dagger$ Hilbert, Werke, vol. 1, p. 276.

$\ddagger$ Pollaczek, Mathematische Zeitschrift, vol. 21 (1924) p. 6. 
3. The Unit $E_{n}$ not an $l^{2}$ th Power. We now consider the units in $k(\zeta)$ when the class number of this field is not prime to $l$. In this case the integer $c$ in (2) might be divisible by $l$; in particular one of the $E$ 's may be the $l$ th power of the unit in $k(\zeta)$.

We shall now show that if

then

$$
r^{l-1} \not \equiv 1\left(\bmod l^{2}\right) \text {, }
$$

$$
E_{n} \neq \rho^{l^{2}}
$$

where $\rho$ is in $k(\zeta)$. Assuming an equality of this type, and using the same method by which, in a previous paper by the writer, the relations (3) and (3a) were handled, * we obtain the following identity in $e^{v}$ :

$$
E_{n}^{l-1}\left(e^{v}\right)=\left(\rho\left(e^{v}\right)\right)^{l^{2}(l-1)}+X\left(e^{v}\right)\left(e^{v l}-1\right)+l j \frac{e^{v l}-1}{e^{v}-1},
$$

where $j$ is a rational integer and $X\left(e^{v}\right)$ is a polynomial in $e^{v}$ with rational integral coefficients. In this expression, taking logarithms and differentiating $2 l$ times, we obtain, using relations (4) and (4a) of the paper last mentioned (p. 620) for $n \neq 1$,

$$
\frac{r^{(l-1)(l-n)}-1}{r^{2 l-2 n}-1} \frac{B_{l}}{2 l}\left(r^{2 l}-1\right) \equiv 0\left(\bmod l^{2}\right) .
$$

Now, since

$$
r^{l-1} \not \equiv 1\left(\bmod l^{2}\right)
$$

then

$$
r^{(l-1)(l-n)}-1
$$

is divisible by $l$ but not by $l^{2}$, which gives a contradiction since $\left(r^{2 l}-1\right)$ and $B_{l} / l$ are prime to $l$.

The University of Texas

\footnotetext{
* Loc. cit., p. 617.
} 